\title{
.
}

\section{ANÁLISIS DE LA RENTABILIDAD ECONÓMICA DEL SISTEMA PORTUARIO ESPAÑOL}

Se analizan los volúmenes de los tráficos de mercancías en los puertos españoles, sus niveles de especialización y los resultados económicos de las autoridades portuarias a lo largo del periodo 2011-2019. El sistema portuario español muestra una gran heterogeneidad derivada de la existencia de puertos grandes, pequeños y medianos, de diferentes grados de orientación y de tráficos cautivos, y de distintos niveles de inserción internacional. Se calcula la rentabilidad en función de la especialización de los tráficos, de la localización de los puertos y de las diferentes estrategias en lo tocante a las tasas portuarias llevadas a cabo por las 28 autoridades portuarias. El trabajo concluye que la especialización en mercancía general, en particular en contenedores, junto al tamaño del puerto, sí son relevantes de cara a obtener una mejor rentabilidad.

Palabras clave: economía marítima, especialización portuaria, gestión portuaria.

Clasificación JEL: D22, D43, L90, R40, R42.

\section{Introducción}

El sistema portuario español (SPE), de titularidad estatal, gestiona más del $65 \%$ de las exportaciones y en torno al $85 \%$ de las importaciones. Los tráficos portuarios son la vía de transporte de más de la mitad del comercio con la Unión Europea y con casi la totalidad de los intercambios comerciales con los terceros países. Está formado por 46 puertos comerciales gestionados por 28 autoridades portuarias (AA PP) mostrando una composición muy diversa. Coexisten puertos grandes, medianos y

* Catedrático de Economía Aplicada. Director del Instituto Universitario de Estudios Marítimos, Universidade da Coruña.

Versión de marzo de 2021.

DOI: https:/doi.org/10.32796/bice.2021.3134.7182 pequeños; y con distintos niveles de especialización. Asimismo se constatan puertos muy dependientes de las industrias próximas a sus recintos; otros muy relacionados con las especificidades de sus hinterlands más cercanos; y unos terceros que efectúan las funciones de transbordo.

El sistema portuario español está caracterizado por ser un servicio multiproducto (vinculado a diferentes tipos de mercancías) que presta un conjunto de servicios heterogéneos, funcionando en un mercado de monopolio natural y en presencia de costes hundidos que actúan de barrera de entrada de potenciales competidores. Funciona en un entorno competitivo imperfecto, con diferentes comportamientos de los actores y bajo una competencia $D$ 
intraportuaria que se ve acentuada por la proximidad de puertos rivales que influyen tanto en la captación y desviación de tráficos como en la oferta de servicios. Los marcos regulatorios puestos en práctica avivaron la competencia interportuaria a la vez que estimularon las bases para lograr ganancias en eficiencia y en la obtención de estímulos de cara a mejorar las posiciones competitivas de cada autoridad portuaria (AP).

Nuestro trabajo se centra en el análisis de los resultados económicos de los puertos españoles a lo largo del periodo 2011-2019. Se Ileva a cabo siguiendo los postulados del Real Decreto Legislativo 2/2011, de 5 de septiembre, donde se explicita que el SPE debe responder a tres principios:

- Autosuficiencia financiera.

- Estar atento a la demanda de los usuarios y ser competitivo.

- Exigencia de obtener una rentabilidad empresarial, cifrada en el $2,5 \%$ anual.

El objetivo radica en comprobar tres hipótesis:

- H.1: si el sistema portuario español, en su conjunto, mantiene tasas de rentabilidad positivas a pesar de su heterogeneidad en el tamaño de los puertos.

- H.2: si dicha rentabilidad portuaria está asociada a los diferentes grados de especialización de los tráficos.

- H.3: si los resultados económicos de los puertos, en función de su autonomía financiera, poseen relación con las diferentes apuestas en torno a los ingresos procedentes de las tasas de utilización y de ocupación.

\section{El modelo de gestión español y revisión de la literatura}

El modelo de gestión portuaria española tiene su origen en la regulación aprobada en 1851 (RD de 17 de diciembre), donde se recoge, por vez primera, la titularidad estatal de algunos puertos españoles, dependiendo del entonces Ministerio de Fomento. Se especifican los puertos de interés general y puertos de interés local. Hubo que esperar un siglo, hasta la aprobación de la Ley 27/1992, de Puertos del Estado y Marina Mercante, para encontrar una nueva definición de la gestión portuaria española. En esta disposición la gestión es conferida a las autoridades portuarias, cuyos objetivos priorizan una mayor eficacia y una mejora en sus servicios y prestaciones portuarias. Se introducen criterios de flexibilidad empresarial y se libera de la rigidez del derecho administrativo procedente de los marcos regulatorios anteriores. Define un único modelo organizativo con amplia autonomía de gestión, pero no profundiza en los conceptos de rentabilidad empresarial. Posteriormente, la Ley 66/1997, que modifica parcialmente la Ley 27/1992, aprueba la libertad tarifaria; y es la Ley 48/2003, de régimen económico y prestación de servicios de los puertos de interés general la que avanza, un poco más, en lo tocante a la flexibilización de las tarifas portuarias, la liberalización de los servicios y la participación de la iniciativa privada. Se menciona el concepto de eficiencia, pero sin una concreción específica del mismo. A partir de la Ley 33/2010, de modificación de la Ley $48 / 2003$, se definen nuevos principios y objetivos del régimen económico del SPE: autofinanciación, fijación de rendimientos razonables y condiciones para hacer frente a la necesidad de nuevas inversiones y devolución de empréstitos. 
Los estudios relacionados con el SPE son muy variados. Se pueden clasificar entre:

- Aquellos que profundizan en los conceptos de productividad y en las relaciones entre los inputs empleados y los outputs obtenidos, para determinar las ventajas competitivas de cada autoridad portuaria (García-Alonso y Martí-Bofarull, 2007; González y Trujillo, 2008).

- Los que analizan la eficiencia y la optimización en lo que concierne a la aplicación de tecnologías, y, en base a ellas, definir un comportamiento más optimizador (Baños-Pino et al., 1999; Coto-Millán et al., 2000).

- Los que se centran en la eficiencia técnica y económica, aportando conclusiones relativas a evaluar los productos, las estructuras de costes y la competitividad (Martínez-Budría et al., 1999; González y Trujillo, 2006; Rodríguez-Álvarez y Tovar, 2012; Fageda y González, 2014).

- Los que evalúan los marcos regulatorios, incidiendo sobre las formas organizativas y de gestión que permiten un funcionamiento más competitivo y eficiente (González y Trujillo, 2008; Castillo-Manzano et al., 2008; Rodríguez-Álvarez y Tovar, 2009; Núñez-Santos y Coto-Millán, 2012; González-Laxe, 2012a).

- Aquellos que evalúan las inversiones y las relaciones con los tráficos, examinando la existencia de relaciones directas o inversas (García-Alonso y Sánchez-Soriano, 2007; González-Laxe, 2012b).

- Los que estudian la sobrecapacidad portuaria, bien por medio de decisiones relativas a las inversiones, bien por las relativas a las estimaciones de demanda
(Baños-Pino et al., 1999; Rodríguez-Álvarez y Tovar, 2012; Tovar y Wall, 2017).

- Los que analizan las captaciones de tráficos y los impactos derivados de los solapamientos en función de las fachadas marítimas españolas (Medal y Sala, 2011; Villaverde y Fernández-Maza, 2012).

Esta clasificación no es, evidentemente, exhaustiva. Simplemente refleja aquellas aportaciones básicas y más relacionadas con los apartados que se abordan en esta contribución. Se han obviado aquellos trabajos que enfatizan los impactos económicos de los puertos en su entorno, con relación a la sostenibilidad ambiental y a su inserción y conectividad marítima, y los vinculados a los servicios portuarios o los relacionados con la problemática laboral y jurídica de los puertos, por citar algunas referencias de investigaciones llevadas a cabo y publicadas en otras revistas.

En el caso español, se produce una amplia disparidad de criterios. Martínez-Budría et al. (1999) subrayaron que los puertos eficientes son los de mayor tamaño, en tanto que los medianos son los menos eficientes. Por el contrario, Coto-Millán et al. (2000) advirtieron de las relaciones inversas entre tamaño y eficiencia, y Bonilla et al. (2002) no encuentran correspondencia entre ambas variables. Sin embargo, sí está definida la relación entre la eficiencia y los cambios en lo tocante a la regulación. Cada nuevo cambio legislativo ha generado efectos positivos en los puertos, llegando a manifestarse tanto en lo que atañe a las ganancias de productividad como en lo referente a los aumentos de cuota de mercado, medidos por la captación y el desvío de tráficos (Castillo-Manzano et al., 2006, 2018; Coto-Millán et al., 2000, 2016). Y tampoco existe duda entre los $D$ 
vínculos existentes entre la evolución de la eficiencia relativa y los hinterlands de los puertos, conduciendo a afirmar que la localización, la integración en las cadenas logísticas y la existencia de hinterlands influencian decididamente en la eficiencia de los puertos (Wiegmans, 2004).

\section{Análisis y resultados de la evolución y especialización de los tráficos portuarios}

El periodo 2011-2019 refleja una tendencia creciente de los tráficos para el conjunto del sistema, apreciándose un mayor aumento en los puertos grandes y medianos. Queda registrada una mayor alza en los puertos especializados en mercancías generales y en aquellos que movilizan los tráficos contenedorizados. Los volúmenes revelan que el crecimiento es más estable en aquellos puertos con fuerte especialización en graneles sólidos o graneles líquidos; es decir, en aquellos cuyos movimientos de mercancías están fidelizados por empresas de refino, plantas de gas, productos químicos o por las demandas de carbón para las centrales térmicas. $Y$, en sentido contrario, se contabilizan crecimientos menores en aquellos puertos con escasa vinculación con industrias próximas al recinto portuario; esto es, con la mercancía convencional.

La diversidad portuaria española se refleja en las distintas cargas movidas en las instalaciones portuarias. Desagregando los tráficos en función de las mercancías y de sus niveles de contenedorización, el sistema portuario español registra lo siguiente:

- Se contabilizan puertos con un alto nivel de especialización (por encima del $45 \%$ del total de sus tráficos) en graneles líquidos (A Coruña, Bilbao, Cartagena, Huelva, Motril y Tarragona).

- Otro grupo con fuerte especialización en graneles sólidos, con ratios superiores al $45 \%$ del total de sus movimientos portuarios (Alicante, Almería, Avilés, Cádiz, Ferrol, Gijón, Málaga, Santander y Sevilla).

- Finalmente, un tercer grupo, con elevado nivel de especialización en mercancía general, por encima del $45 \%$ de sus tráficos totales (Alicante, Algeciras, $\triangleright$

CUADRO 1

EVOLUCIÓN DEL TRÁFICO PORTUARIO ESPAÑOL SEGÚN PRESENTACIÓN DE LAS CARGAS

(Millones de toneladas)

\begin{tabular}{|c|c|c|c|c|c|}
\hline \multirow{2}{*}{ Año } & Graneles líquidos & Graneles sólidos & \multicolumn{2}{|c|}{ Mercancía general } & \multirow{2}{*}{ Total } \\
\cline { 3 - 5 } & & & Mercancía convencional & Mercancía contenedorizada & \\
\hline 2011 & 150,8 & 79,3 & 55,7 & 157,9 & 443,7 \\
2012 & 153,6 & 80,4 & 56,9 & 162,1 & 461,0 \\
2013 & 152,0 & 80,3 & 58,4 & 155,0 & 445,7 \\
2014 & 160,7 & 89,6 & 62,3 & 155,5 & 468,1 \\
2015 & 168,0 & 96,0 & 65,1 & 159,3 & 488,4 \\
2016 & 167,6 & 92,0 & 67,7 & 168,3 & 495,6 \\
2017 & 178,2 & 101,4 & 71,0 & 181,4 & 532,1 \\
2018 & 180,7 & 102,4 & 76,3 & 191,0 & 550,4 \\
2019 & 187,1 & 90,8 & 78,7 & 195,3 & 551,9 \\
\hline
\end{tabular}


Baleares, Barcelona, Ceuta, Las Palmas, Málaga, Marín, Melilla, Pasaia, S. C. de Tenerife, Valencia, Vigo y Vilagarcía).

Este mosaico se complementa con los diferentes niveles de contenedorización.

Se distinguen tres niveles de puertos:

- Los poseedores de un alto nivel de inserción internacional y elevados volúmenes de tráfico, superando los 500.000 TEUS $^{1}$ anuales (Algeciras, Valencia, Barcelona, Bilbao, Las Palmas y S. C. de Tenerife).

- Los que contabilizan entre los 100.000 y 500.000 TEUS anuales (Vigo, Castellón, Alicante, Sevilla, Málaga y Baleares).

- Los demás puertos, todos ellos por debajo de 100.000 TEUS anuales.

El análisis de la superficie concesionada en los recintos portuarios detecta nuevas diferencias entre las autoridades portuarias. Dicho índice revela la capacidad de cesión a las empresas privadas de un espacio de dominio público dedicado a la utilización y manipulación de mercancías específicas. Se entiende que un mayor nivel de superficie concesionada es sinónimo de haber atraído inversiones y mejora del nivel de aprovechamiento y eficacia de los recintos portuarios. Atendiendo a esta concepción, el SPE muestra distintos grados de utilización y uso. Destacan, por sus elevados niveles, aquellos puertos con índices superiores al $70 \%$ de superficie concesionada en sus recintos (Marín, 94\%; Tarragona, 93\%; Bilbao, 85\%; Ceuta, 85\%; Málaga, 84\%; Barcelona, 81\%; Pasaia, $81 \%$; S. C. de Tenerife, $77 \%$; Cartagena, $76 \%$; Ferrol, 76\%; Valencia, 74\%; y Vigo, 72\%). También sobresalen, en sentido contrario, por

Siglas de twenty-foot equivalent units, equivalentes a 20 pies. su escaso nivel de concesión del recinto portuario, con ratios inferiores al $45 \%$, las autoridades de Baleares, 40\%; Melilla, 41\%; Las Palmas, $41 \%$; Castellón, 42\%; A Coruña, 44\%; y Almería, $44 \%$; ya sea por disponer de mucha superficie portuaria o por estar muy concentrados los movimientos portuarios en tráficos muy especializados. La tendencia experimentada en los últimos años (periodo 2011-2019) revela un incremento de dicho porcentaje (del $38 \%$ al $65 \%$ ); y ello ha supuesto avances muy notables en puertos de Cartagena, Marín, Tarragona, Vigo, Huelva, Barcelona, Motril, Pasaia, Málaga, Valencia, Algeciras o Cádiz. Por el contrario, el porcentaje de la superficie concesionada ha retrocedido en los últimos años en los puertos de Vilagarcía y Las Palmas.

\section{Análisis y resultados de los ingresos y resultados económicos del SPE}

Los ingresos del SPE se componen, principalmente, de las tasas referidas a la ocupación, a la actividad, a las ayudas a la navegación y a la utilización; siendo esta última aquella que comprende las tasas a los buques, a las embarcaciones deportivas y de recreo, al pasaje, a la mercancía, a la pesca fresca y a la utilización de zonas de tránsito, tal y como exponemos en el Cuadro 2. Conceptualmente, la tasa de ocupación hace referencia a la ocupación privativa del dominio público portuario. La tasa de actividad se refiere al ejercicio de la actividad comercial, industrial y de servicios en el dominio público portuario. La tasa de utilización grava la utilización especial de las instalaciones portuarias, que, a su vez, se subdivide en la tasa del buque, la tasa al pasaje, la tasa a la mercancía, la tasa de la pesca fresca, la $D$ 
tasa a las embarcaciones deportivas y de recreo, y las de utilización especial de las zonas de tránsito. Por último, la tasa de ayuda a la navegación se refiere a los gravámenes por los servicios de señalización marítima. Esto es, la tasa de ocupación es un abono que las empresas efectúan por el alquiler o cesión de un espacio que ocupan en el recinto portuario, siendo, en consecuencia, un ingreso fijo para la autoridad portuaria; en tanto que las tasas de actividad y de utilización son variables, en la medida que se abonan en función de los movimientos de buques, mercancías o pasaje.

CUADRO 2

DESGLOSE DE LAS TASAS PORTUARIAS EN EL SISTEMA PORTUARIO ESPAÑOL

\begin{tabular}{|l|l|l|}
\hline \multicolumn{1}{|c|}{ Tipo } & \multicolumn{1}{|c|}{ Concepto } & \multicolumn{1}{c|}{$\begin{array}{c}\text { Tasas } \\
\text { aplicadas }\end{array}$} \\
\hline \multirow{2}{*}{$\begin{array}{l}\text { Tasa de utilización } \\
\text { (buques) }\end{array}$} & $\begin{array}{l}\text { Utilización especial de las } \\
\text { instalaciones portuarias }\end{array}$ & Buque \\
\cline { 3 - 3 } & Pasaje \\
\cline { 2 - 3 } \multirow{2}{*}{$\begin{array}{l}\text { Tasa a empresas } \\
\text { concesionarias }\end{array}$} & $\begin{array}{l}\text { Ocupación privativa del } \\
\text { dominio público }\end{array}$ & $\begin{array}{l}\text { Tasa } \\
\text { ocupación }\end{array}$ \\
\cline { 2 - 3 } & $\begin{array}{l}\text { Ejercicio en el dominio } \\
\text { publico de actividades } \\
\text { comerciales y de servicios }\end{array}$ & $\begin{array}{l}\text { Tasa } \\
\text { actividad }\end{array}$ \\
\hline \multirow{2}{*}{ Fuente: elaboración propia. } & \multicolumn{2}{|l}{} \\
\hline
\end{tabular}

La suma, para el año 2019, de los importes de las tasas de ocupación (286 millones de euros) y utilización (604 millones de euros) suponen el $85 \%$ de los ingresos obtenidos por las tasas portuarias del SPE. A continuación se sitúa la tasa de actividad (134 millones de euros). Y, dentro de las tasas de utilización, las correspondientes al buque (244 millones de euros) y a las mercancías (262 millones de euros) ascienden al $48 \%$ del total de los ingresos por las tasas.

Al referirnos a las tres principales fuentes de ingresos (esto es, a las tasas de ocupación, mercancía y buque), el SPE revela que hay autoridades portuarias que apuestan por estrategias referenciadas a las tasas de ocupación (porcentajes superiores al 30\%), como Sevilla (35\%), Barcelona (32\%), Bilbao (31\%) y Alicante (30\%). Otras se muestran más favorables a enfocar sus ingresos sobre las tasas a las mercancías; son los casos de Cartagena (39\%), A Coruña (35\%), Valencia (34\%), Bilbao (32\%), Vigo (31\%) y Huelva (30\%). Y, finalmente, unos terceros hacen hincapié en que la parte sustancial de sus ingresos se basa en las tasas al buque: son los ejemplos de Cádiz (32\%), Gijón (30\%), Ferrol (30\%) y los puertos canarios de Las Palmas (33\%) y S. C. de Tenerife (28\%).

Es notable, en consecuencia, la variedad existente en lo que atañe a los ingresos medios por dichas tasas (tal y como se expone en el Cuadro 3). Las razones de dichas diferencias se deben a las distintas orientaciones definidas en los planes de empresa de cada una de las autoridades portuarias. Sobresale el hecho de cómo las tasas medias más elevadas sobre los buques se registran en Avilés, Gijón, Ferrol y Marín, en tanto que las menores se llevan a cabo en Algeciras, Baleares, Melilla, S. C. de Tenerife y Málaga. En lo que compete a las tasas medias sobre las mercancías destacan las posiciones elevadas de los puertos de Vilagarcía, Vigo, Marín y Melilla. Y en lo que hace referencia a las horquillas más bajas se sitúan los puertos de Algeciras, Almería, Ferrol y Castellón. En lo que hace mención a la relevancia que supone la tasa de ocupación sobre los ingresos de los puertos, el ingreso medio por dicha tasa es elevado en Baleares, A Coruña, Pasaia, Gijón, Barcelona, Málaga y Bilbao, en tanto que los porcentajes más bajos corresponden a los puertos de Ferrol, Huelva, Cádiz, Sevilla, Almería y Castellón. Dicha variabilidad es la consecuencia de una decisión estratégica de cada autoridad portuaria, determinada por sus propios condicionantes intrínsecos $D$ 
derivados de la localización, de los competidores, de la industria asociada al puerto y de los tráficos de transbordo.

Los ingresos obtenidos por el sistema portuario español muestran una tendencia creciente. El importe neto de la cifra de negocios del SPE ascendió, en 2019, a un total de 1.157 millones de euros, cifra superior a la registrada en años anteriores. En lo que atañe a los importes de la cifra de negocios por AP, el ranking lo encabeza Barcelona (173,5 millones de euros), seguido de Valencia (140,3 millones de euros) y Algeciras (83,5 millones de euros). Dichas autoridades portuarias suman cerca de 400 millones, equivalente al $35 \%$ del total. A continuación se sitúa un grupo de puertos medianos, que incluye a Baleares $(78,5)$, Las Palmas $(77,6)$, Bilbao $(70,5)$, Tarragona $(56,4)$, S. C. de Tenerife $(46,3)$, Huelva $(45,3)$, Cartagena $(43,2)$ y Gijón $(42,1)$. Con más de 10 y $\triangleright$

\section{CUADRO 3}

INGRESOS Y RESULTADOS DE LAS AUTORIDADES PORTUARIAS EN 2019

\begin{tabular}{|c|c|c|c|c|c|}
\hline \multirow{2}{*}{$\begin{array}{l}\text { Autoridad } \\
\text { portuaria }\end{array}$} & \multicolumn{3}{|c|}{$\begin{array}{l}\text { Porcentaje que representan sobre el importe total } \\
\text { de las tasas }\end{array}$} & \multirow{2}{*}{$\begin{array}{l}\text { Importe neto cifra } \\
\text { negocios (miles de euros) }\end{array}$} & \multirow{2}{*}{$\begin{array}{l}\text { Resultado final } \\
\text { (miles de euros) }\end{array}$} \\
\hline & Tasa ocupación & Tasa buque & Tasa mercancía & & \\
\hline A Coruña ................... & 24,9 & 31,3 & 33,7 & 28.870 & 1.042 \\
\hline Alicante ...................... & 33,8 & 16,5 & 15,4 & 12.176 & 2.299 \\
\hline 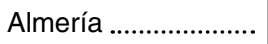 & 16,9 & 18,3 & 16,1 & 15.469 & 1.870 \\
\hline 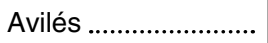 & 29,2 & 28,3 & 27,0 & 16.562 & 4.005 \\
\hline Bahía de Algeciras. & 17,6 & 13,5 & 22,4 & 83.732 & 19.783 \\
\hline Bahía de Cádiz ......... & 25,0 & 35,1 & 15,7 & 20.573 & 2.412 \\
\hline Baleares ..................... & 32,8 & 14,3 & 14,4 & 84.740 & 28.303 \\
\hline Barcelona .................... & 36,3 & 21,2 & 23,9 & 172.433 & 44.164 \\
\hline Bilbao ............................... & 34,1 & 22,4 & 32,0 & 69.277 & 7.551 \\
\hline Cartagena .... & 11,1 & 31,4 & 39,5 & 47.398 & 28.054 \\
\hline Castellón ................... & 24,8 & 23,5 & 31,4 & 31.070 & 9.947 \\
\hline Ceuta .......................... & 21,5 & 7,1 & 7,3 & 16.031 & 1.119 \\
\hline Ferrol ............. & 23,3 & 28,1 & 26,5 & 17.754 & 3.684 \\
\hline Gijón ......................... & 32,6 & 31,4 & 23,0 & 37.861 & 5.034 \\
\hline Huelva ......................... & 22,9 & 23,8 & 38,9 & 45.130 & 10.475 \\
\hline Las Palmas ............... & 30,2 & 32,5 & 12,4 & 80.997 & 42.333 \\
\hline Málaga ....................... & 31,8 & 31,3 & 10,6 & 19.505 & 2.597 \\
\hline Marín .............................. & 28,8 & 22,1 & 29,6 & 9.579 & 1.327 \\
\hline Melilla ........................... & 26,7 & 14,6 & 10,4 & 9.180 & -2.909 \\
\hline Motril ............. & 18,9 & 30,9 & 31,3 & 7.780 & 1.476 \\
\hline 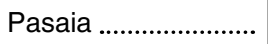 & 30,0 & 14,6 & 22,1 & 11.894 & 750 \\
\hline S. C. de Tenerife ...... & 23,4 & 26,8 & 16,9 & 47.333 & 15.715 \\
\hline Santander .................... & 34,4 & 26,0 & 21,4 & 22.981 & 3.641 \\
\hline Sevilla ......................... & 38,6 & 10,1 & 17,6 & 19.884 & 1.706 \\
\hline Tarragona ................. & 25,9 & 29,7 & 28,0 & 57.650 & 3.533 \\
\hline Valencia ........................ & 22,1 & 23,8 & 37,3 & 139.771 & 24.368 \\
\hline Vigo ............................... & 30,1 & 20,3 & 19,0 & 26.459 & 1.405 \\
\hline Vilagarcía ....... & 35,0 & 18,2 & 33,8 & 5.445 & 1.155 \\
\hline Total .............................. & 27,6 & 23,5 & 25,2 & 1.157.532 & 276.878 \\
\hline
\end{tabular}


menos de 40 millones de euros de cifra de negocios hay catorce puertos. Finalmente, el cuarto nivel viene representado por aquellas AA PP que contabilizan una menor cifra de negocios, inferior a 10 millones de euros: son Vilagarcía (5), Motril $(7,7)$, Marín $(9,7)$ y Melilla $(9,7)$.

Los beneficios obtenidos por el sistema portuario español son elevados. En el año 2019 sumaron un total de 276 millones de euros. Si a la cifra de negocios final le restamos los gastos de personal, de explotación, las amortizaciones del inmovilizado, el deterioro por enajenación del inmovilizado, los gastos y los resultados financieros, obtendremos el resultado final del periodo. Las cifras de esta última rúbrica dejan ver la amplia disparidad interportuaria en España, tal y como se refleja en el Cuadro 3. Solo una autoridad portuaria presenta resultados negativos en el año 2019 (Melilla) y otra muestra resultados económicos menores de un millón de euros (Pasaia). Quince AA PP registran resultados económicos entre un millón y 5 millones positivos (A Coruña, Alicante, Almería, Avilés, Cádiz, Ceuta, Ferrol, Málaga, Marín, Motril, Santader, Sevilla, Tarragona, Vigo y Vilagarcía). Tres anotan beneficios comprendidos entre 5 y 10 millones de euros (Bilbao, Castellón y Gijón). $\mathrm{Y}$, finalmente, un total de ocho autoridades portuarias contabilizan beneficios superiores a los 10 millones de euros. Son, por volumen, de mayor a menor: Barcelona $(44,1)$, Las Palmas $(42,3)$, Baleares $(28,3)$, Cartagena (28), Valencia $(24,3)$, Algeciras $(19,7)$, S. C. de Tenerife $(15,7)$ y Huelva $(10,4)$.

\section{Análisis y resultados de la rentabilidad portuaria}

La rentabilidad anual, a efectos de la Ley de Puertos 33/2010 (y luego artículo 157 del Texto
Refundido, RDL 2/2011), se refiere al cociente entre: a) el resultado del ejercicio después de impuestos, excluyendo del mismo el deterioro y el resultado por enajenaciones del inmovilizado y otros resultados que tengan el carácter de extraordinario, así como los ingresos financieros correspondientes a la incorporación al activo de gastos financieros y el saldo del fondo de compensación interportuario aportado o recibido; y b) el activo no corriente neto medio del ejercicio excluyendo el inmovilizado en curso, el inmovilizado correspondiente a terrenos y bienes naturales sobre los que no se haya desarrollado ningún tipo de actividad durante el ejercicio, los activos por impuestos diferidos y los deudores comerciales no comerciales. A los efectos de los cálculos, la incorporación de una nueva infraestructura portuaria básica (dique de abrigo, esclusas y accesos marítimos) se prorratea durante siete años desde la fecha del acta de recepción.

Corresponde a cada AP, según la normativa vigente, fijar la rentabilidad anual atendiendo a las características específicas del puerto, a las diferentes situaciones competitivas, a la previsión de la demanda y a las condiciones de alejamiento e insularidad. Así mismo, cada AP puede modular el importe de las tasas y tarifas para obtener los ingresos necesarios en la consecución de la rentabilidad del sistema. Existe, por tanto, un principio de libertad tarifaria, pero con límites. No pueden ser inferiores al coste de los servicios, y, por lo tanto, se estima el principio de cobertura de los costes.

En la Ley $33 / 2010$ se ha fijado como compromiso referencial obtener una rentabilidad anual del 2,5\%. El balance del periodo 20112019 permite evaluar el comportamiento y la capacidad de gestión de cada una de las 28 autoridades portuarias. En primer término, el conjunto muestra una tendencia parecida a $\triangleright$ 
la de una sonrisa. El primer año de aplicación de la Ley 33/2010 recoge un rendimiento del $1,1 \%$, para subir, inmediatamente, al 2,34\% en el año 2012. Luego de un periodo de descenso, que tiene como punto bajo el año 2015 con el $1,70 \%$, comienza una fase de alza de la rentabilidad, y se sitúa progresivamente en el año 2018 en el 2,52\% y en 2019 en el 2,43\%; esto es, dentro de la horquilla estimada en el RD Legislativo de 2/2011.

El comportamiento no ha sido el mismo para todas las autoridades portuarias (véase Cuadro 4). Se registra:

- Rentabilidad con tendencia negativa o con resultados negativos: A Coruña, Ceuta, Melilla y Vilagarcía.

- Con tendencia positiva y recuperando situaciones de resultados negativos: Alicante, Almería, Gijón, Marín, Motril, Pasaia, S. C. de Tenerife y Santander.

- Con tendencia positiva y muy estable: Cádiz, Bilbao, Tarragona y Vigo.

- Con tendencia estable dentro de una alta rentabilidad: Avilés, Algeciras, Barcelona, Baleares, Cartagena, Castellón, Huelva, Las Palmas y Valencia.

- Con tendencia positiva, pero con amplias fluctuaciones durante el periodo de recuperación de tráficos: Ferrol, Málaga y Sevilla.

\section{Discusión}

La normativa española referida al funcionamiento de las AA PP se sustentó sobre cuatro pilares. Introduce una flexibilización en lo referente al modelo tarifario, de manera que cada AP puede fijar sus propias tasas y adaptarse a la realidad económica de cada momento. En segundo término, se avanzó en la liberalización de los servicios portuarios y de las actividades económicas y comerciales mediante el otorgamiento de concesiones y asignación de los servicios técnico-náuticos (amarradores, practicaje, remolcadores y servicios de recogida de residuos). En tercer lugar, refuerza el principio de autosuficiencia económica, presente desde la Ley 27/1992, por el que las AA PP recaudan las tasas por la utilización de sus instalaciones sin tener que depender de los fondos aportados por los Presupuestos Generales del Estado. Finalmente, se establece como objetivo del SPE alcanzar una rentabilidad global del sistema del 2,5\% anual. En consecuencia, las autoridades portuarias han de saber ajustar las tasas al entorno de su competencia, toda vez que cuatro quintas partes de los ingresos están relacionados con la explotación procedente de las mencionadas tasas portuarias y con ellas poder abordar los costes de explotación y aumentar la capacidad para financiar la inversión y cubrir las amortizaciones que necesita una AA PP.

La contribución de esta aportación subraya lo siguiente. Se registra una estrecha correlación entre el volumen de tráficos movilizados en un puerto con las tasas que se cobran. Es decir, a medida que se incrementa la carga (aparentemente, sin relación al tipo de la misma) se consiguen mayores niveles de ingresos. Y esta dinámica hace a las AA PP más competitivas frente a posibles rivales, puesto que un incremento de los tráficos permite reducir los costes unitarios y beneficiarse de las economías de escala.

Los ingresos portuarios están muy relacionados tanto con los índices de especialización en graneles como con los volúmenes de tráficos contenedorizados. Los ingresos y la rentabilidad de las AA PP están vinculados, $D$ 
CUADRO 4

EVOLUCIÓN DE LA RENTABILIDAD ANUAL DE LAS AUTORIDADES PORTUARIAS

(En porcentaje)

\begin{tabular}{|c|c|c|c|c|c|c|c|c|c|}
\hline Autoridad portuaria & 2011 & 2012 & 2013 & 2014 & 2015 & 2016 & 2017 & 2018 & 2019 \\
\hline 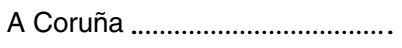 & 1,14 & 4,10 & 2,11 & 0,87 & 1,05 & 0,79 & 0,71 & 0,50 & 0,05 \\
\hline Alicante & $-0,84$ & $-0,81$ & $-0,47$ & $-0,53$ & $-0,01$ & 0,79 & 0,81 & 1,34 & 1,46 \\
\hline Almería ............. & 0,14 & 0,00 & $-0,17$ & 0,51 & 0,39 & 0,51 & 1,94 & 1,77 & 1,51 \\
\hline 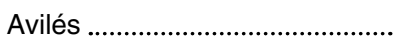 & 2,43 & 2,57 & 2,57 & 1,37 & 2,50 & 2,43 & 2,57 & 2,75 & 3,08 \\
\hline 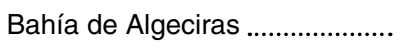 & 4,77 & 5,10 & 5,47 & 3,68 & 3,37 & 3,38 & 2,63 & 3,44 & 3,36 \\
\hline 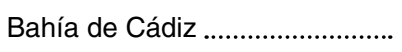 & 0,59 & 1,24 & 0,54 & 0,13 & 0,47 & 0,97 & 0,03 & 0,18 & 0,55 \\
\hline 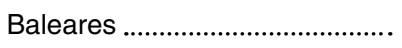 & 4,21 & 4,20 & 6,32 & 4,14 & 5,30 & 4,67 & 4,88 & 6,73 & 6,93 \\
\hline 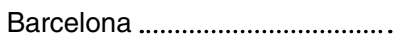 & 3,68 & 3,80 & 3,75 & 2,87 & 2,32 & 2,38 & 3,14 & 3,35 & 2,95 \\
\hline 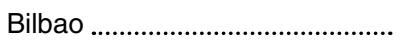 & 1,15 & 0,94 & 0,80 & 0,63 & 0,96 & 0,89 & 1,42 & 1,45 & 1,15 \\
\hline Cartagena .. & 6,89 & 10,92 & 8,96 & 9,40 & 7,95 & 7,13 & 7,92 & 7,31 & 8,37 \\
\hline 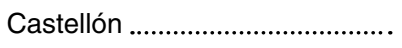 & 1,31 & 1,33 & 2,06 & 2,18 & 3,09 & 3,09 & 4,56 & 5,15 & 4,84 \\
\hline Ceuta & $-4,09$ & $-1,21$ & 0,60 & $-1,47$ & $-2,72$ & $-1,05$ & $-3,88$ & $-0,77$ & $-1,65$ \\
\hline 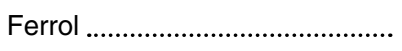 & 2,43 & 3,12 & 3,13 & 2,75 & 1,37 & 2,00 & 2,80 & 3,08 & 1,92 \\
\hline 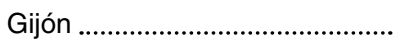 & $-1,94$ & 0,80 & 0,49 & 0,26 & 0,53 & 0,35 & 1,62 & 0,41 & 0,66 \\
\hline 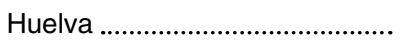 & 2,55 & 3,70 & 3,69 & 3,61 & 3,40 & 3,31 & 2,84 & 3,19 & 2,78 \\
\hline 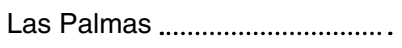 & $-0,60$ & 1,60 & 2,22 & 1,43 & 2,39 & 2,50 & 2,16 & 4,61 & 6,61 \\
\hline 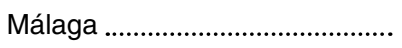 & 1,50 & $-1,24$ & $-0,91$ & 0,13 & $-0,74$ & 0,84 & 0,82 & 1,27 & 1,65 \\
\hline Marín & 2,61 & 4,12 & 2,80 & 1,11 & 2,26 & 2,89 & 3,05 & 2,52 & 1,81 \\
\hline 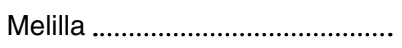 & $-3,14$ & $-1,27$ & $-1,08$ & $-1,79$ & $-1,98$ & $-1,08$ & $-1,77$ & $-2,50$ & $-3,40$ \\
\hline 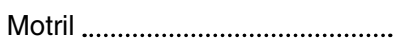 & $-0,33$ & $-0,31$ & 0,15 & $-1,78$ & 0,71 & 0,60 & 1,36 & 1,85 & 0,44 \\
\hline Pasaia & 0,48 & 1,20 & 1,69 & 2,52 & 0,88 & 0,98 & $-0,25$ & $-0,20$ & 0,62 \\
\hline 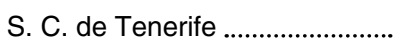 & $-1,88$ & $-0,83$ & 0,51 & $-0,01$ & 0,89 & 0,94 & 3,11 & 2,70 & 1,75 \\
\hline 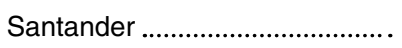 & 0,93 & 0,31 & 0,51 & 0,34 & 0,13 & 0,22 & 0,65 & 1,89 & 1,34 \\
\hline Sevilla .................................................... & 2,14 & 1,15 & $-1,06$ & $-0,48$ & $-0,34$ & 0,67 & 0,16 & $-0,06$ & 0,09 \\
\hline 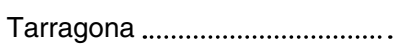 & 4,00 & 3,55 & 1,40 & 1,66 & 1,74 & 1,80 & 2,83 & 2,26 & 1,44 \\
\hline 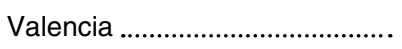 & 1,91 & 2,04 & 1,45 & 1,93 & 1,13 & 2,96 & 3,40 & 3,12 & 2,53 \\
\hline 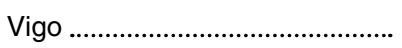 & 0,36 & 0,54 & 0,50 & $-0,20$ & $-0,61$ & 0,25 & 0,49 & 2,82 & 0,79 \\
\hline 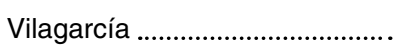 & $-1,80$ & $-1,16$ & $-2,12$ & $-1,83$ & $-2,16$ & $-1,87$ & $-0,76$ & $-1,07$ & $-0,25$ \\
\hline 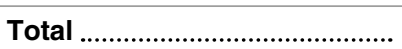 & 1,71 & 2,34 & 2,22 & 1,79 & 1,70 & 2,17 & 2,47 & 2,52 & 2,43 \\
\hline
\end{tabular}

mayormente, con los mayores volúmenes de tráficos contenedorizados; esto es, con aquellos puertos que poseen una amplia frecuencia de escalas de buques y en los que la presencia de compañías de líneas regulares es notable, coincidiendo con lo expuesto por González y Trujillo (2009).

Los puertos con mayores niveles de tráfico contenedorizado poseen elevados índices de superficie concesionada. Se constata, igualmente, aunque no es generalizada, que dicha característica también se manifiesta en puertos con terminales dedicadas a graneles (bien líquidos o bien sólidos), en los que se supone la existencia de un tráfico muy condicionado (son los casos de los puertos petroleros o carboneros, próximos a una refinería o una central térmica de carbón, respectivamente). Muy excepcionalmente, existen puertos que muestran un pequeño volumen de tráfico, pero con elevados índices de superficie concesionada y altos índices de rentabilidad. La existencia de $D$ 
mayor superficie concesionada influye en la rentabilidad portuaria. Dicha relación es ambivalente en la medida que un incremento de la presencia privada en el puerto podría llegar a estimular el hecho de un menor rendimiento económico de la AP. La razón estriba en que la autoridad portuaria, al buscar el equilibrio entre los ingresos fijos anuales por ocupación y los variables por el uso, puede llegar a variar sus decisiones estratégicas y proceder a bonificar ciertas tasas a los usuarios más frecuentes con el objeto de fidelizarlos, o bien podría producirse una deducción en las tasas debida a la presión negociadora que aquellos ejercen sobre los responsables de los puertos.

De esta forma, varias AA PP (Barcelona, Gijón, Alicante, Baleares, Bilbao, Vilagarcía) priorizan los ingresos por ocupación; esto es, buscan asegurar ingresos fijos y proporcionar a sus usuarios (importantes empresas ubicadas en el entorno portuario) relevantes descuentos que podrían resultar ventajosos para fidelizar determinados volúmenes de cargas y reforzar el posicionamiento geográfico de determinadas instalaciones. Ello no quiere decir, de manera inexorable, que mayores tasas de ocupación supongan incrementar la rentabilidad de las autoridades portuarias, porque existen otras, con elevados ingresos por tasa de ocupación, que también utilizan las tasas a las mercancías como soporte de los ingresos. De esta manera, lo óptimo sería, por un lado, fidelizar los propios tráficos a lo largo del tiempo y lograr, simultáneamente, poseer altos porcentajes de ingresos procedentes de las tasas de ocupación.

Decisiones como las de basar la rentabilidad de un puerto sobre los ingresos procedentes de las tasas a la mercancía no resulta suficiente para garantizar una elevada rentabilidad, aunque sí contribuye a lograrlo. Existen varias explicaciones dada la amplia dispersión de casos. Los que consiguen una mayor rotación de tráficos en los muelles se consideran los más eficientes, y los que poseen mayores niveles de tráficos internacionales son los que muestran mayores facilidades para generar ingresos. Así mismo, se contempla la existencia de una linealidad entre los tráficos de mercancía general y graneles con el consumo y producción nacional.

El análisis de las tasas portuarias se convierte en una pieza clave a la hora de alcanzar ratios de rentabilidad positivos y crecientes. De una parte, la cuota íntegra de la tasa portuaria a la mercancía puede presentar una amplia variabilidad (García y Pardo, 2012). Es decir, pueden ser distintas puerto por puerto; $y$, también, por tráficos, pues depende de cómo se lleven a cabo los movimientos de mercancías. Así pues, está en función de si existen concesiones o no en el recinto portuario, de si nos referimos a mercancías embarcadas o desembarcadas, de si se registran transbordos o no, o de si las mercancías salen o no del recinto portuario por vía férrea, entre las principales opciones.

El análisis de la existencia de economías de escala, y en consecuencia, el decrecimiento de los costes unitarios, resulta básico en las estrategias comerciales. La especialización permite captar más mercancías, desviar tráficos y acelerar las capacidades de captura de mercados. En tanto que el tamaño de los puertos incide a la hora de la elección de una posición hipotéticamente ventajosa. En definitiva, atendiendo a la amplia pluralidad de puertos a lo largo del perímetro costero español, las AA PP se ven obligadas a subrayar sus factores de atractividad y a diseñar correctamente sus factores de caracterización (Felicio et al., 2014; González-Laxe et al., 2015). En este sentido, aquellas AA PP con tráficos de transbordo son más proclives a obtener tasas de rentabilidad más altas. 


\section{Conclusiones}

La dimensión y las características de un puerto influyen de manera muy específica sobre la operatividad y la rentabilidad. Aunque esta relación encierra ciertas contradicciones en lo que respecta a la eficiencia. Sin embargo, la eficiencia de los puertos aumenta con la dimensión, demostrando la significatividad de las economías de escala, la capacidad de aumentar los factores de atracción y los efectos hub. La vinculación con los rasgos del hinterland, debido a sus mayores o menores efectos económicos, influye en los niveles de eficiencia financiera.

Se verifica la relevancia que posee la especialización del puerto en función de sus cargas. Normalmente, los mayores niveles de eficiencia se registran en los que predomina la carga contenedorizada, aunque también se contabilizan en aquellos con intensos niveles de especialización en graneles, en la medida que explican la elevada rentabilidad por medio de unos tráficos muy condicionados y fidelizados a las empresas localizadas en el puerto o próximos al mismo. También, la especialización afecta al desempeño de los puertos a través de los servicios ofertados y de las infraestructuras disponibles. Esto es, a mayor oferta de servicios marítimos, mayor será la capacidad de atracción de tráficos en el puerto. Tanto la competencia interportuaria como sus niveles de especialización poseen un fuerte impacto sobre la eficiencia, desempeño y competitividad. Los efectos de las economías de escala asociadas a los movimientos de mercancías son determinantes en los niveles de rentabilidad portuaria.

Este trabajo tiene implicaciones en la gestión de los puertos debido a las distintas posibilidades a la hora de medir la influencia de las características y variables clave en lo que a la gestión operativa se refiere, servir para una más eficiente adecuación del modelo de gobernanza y contribuir a vislumbrar las mejores opciones de un desarrollo comercial y la definición de acciones relativas a las inversiones. En suma, los decisores públicos deben tener en consideración muchos aspectos a la hora de determinar sus apuestas estratégicas.

\section{Bibliografía}

Baños-Pino, J., Coto-Millán, P., y Rodríguez-Álvarez, A. (1999). Allocative efficiency and overcapacity: an application. International Journal of Transport Economics, 26, 181-199.

Bonilla, M., Casasús, T., Medal, A., y Sala, R. (2002). Traffic in the Spanish Ports: an efficiency analysis. International Journal of Transport Economics, XXIX (2), 215-230.

Castillo-Manzano, J. I., Castro-Nuño, M., González-Laxe, F., y Pedregal, D. (2018). Legal reform and the devolution of the Spanish Ports System: an econometric assessment. Utilities Policy, 50(C), 73-82.

Castillo-Manzano, J. I., López-Valpuesta, L., y Pérez-Garcia, J. (2006). Análisis de las Leyes de 1992 y 1997 sobre el sistema portuario español. Boletín Económico de ICE, (2871), 47-57.

Castillo-Manzano, J. I., López-Valpuesta, L., y Pérez-García, J. (2008). Economic Analysis of the Spanish Port sector reform during the 1990s. Transportation Research Part A: Policy and Practice, 42(8), 1056-1063.

Coto-Millán, P., Baños-Pino, J. L., y Rodríguez-Álvarez, A. (2000). Economic efficiency in Spanish Ports: some empirical evidence. Maritime Policy and Management, 27(2), 169-174.

Coto Millán, P., Fernández, X. L., Hidalgo, S., y Pesquera, M. A. (2016). Public regulation and technical efficiency in the Spanish Port Authorities: 1986-2012. Transport Policy, 47, 139-148. 
Fageda, X., y González-Aregall, M. (2014). Port charges in Spain: the roles of regulation and market forces. International Journal of Shipping and Transport Logistics, 6(2), 152-171.

Felicio, J. A., Caldeirinha, V., y Dionisio, A. (2014). The effects of port and container terminal: characteristics on terminal perfomance. Maritime Economics and Logistics, 17(4), 493-514.

García, A. M., y Pardo, A. (2012). La tasa de la mercancía en la Ley de Puertos: aplicación a sectores económicos estratégicos. Papeles de Economía Española, (131), 164-178.

García-Alonso, L., y Marti-Bofarull, M. (2007). Impact of port investment on efficiency and capacity to attract traffic in Spain: Bilbao vs. Valencia. Maritime Economics and Logistic, 9(3), 254-267.

García-Alonso, L., y Sánchez-Soriano, J. (2007). Evolución de las inversiones vs. Evolución de la selección portuaria provincial. Investigaciones Regionales, (11), 113-128.

González, M. M., y Trujillo, L. (2006). La medición de la eficiencia en el sector portuario: revisión de la evidencia empírica (Documento de Trabajo 2005-06). Universidad de Las Palmas. Dpto. de Análisis Económico Aplicado.

González, M. M., y Trujillo, L. (2008). Reforms and infrastructure efficiency in Spain's container ports. Transportation Research Part A: Policy and Practice, 42(1), 243-257.

González, M. M., y Trujillo, L. (2009). Efficiency measuring in the port industry: a survey of the empirical evidence. Journal of Transport Economics and Policy, 43(2), 157-192.

González-Laxe, F. (2012a). El marco regulatorio de los puertos españoles: resultados y conectividad internacional. Economía Industrial, (386), 27-38. https://www.mincotur.gob.es/Publicaciones/Publicacionesperiodicas/Economialndustrial/RevistaEconomialndustrial/386/Fernando $\% 20$ Gonz\%C3\%A1lez\%20Laxe.pdf
González-Laxe, F. (2012b). Inversiones y tráfico portuario: un análisis de la realidad española. Boletín FAL/CEPAL, 313 (9). https://repositorio.cepal. org/bitstream/handle/11362/36185/1/FAL_Bolet \%C3\%ADn313_es.pdf

González-Laxe, F., Freire, M. J., y Pais, C. (2015). Port Policy and Port Choice: The Spanish case. International Journal of Transport Economics, 42(4), 529-553.

Martínez-Budría, E., Diaz-Armas, R., Navarro, M., y Ravelo-Mesa, T. (1999). A study of the efficiency of Spanish Ports Authorities using data envelopment analysis. International Journal of Transport Economics, XXVI (2), 237-253.

Medal, A., y Sala, R. (2011). Análisis de la eficiencia y liderazgo de los puertos españoles por áreas geográficas. Revista de Estudios Regionales, (91), 161-182. http://www.revistaestudiosregionales.com/documentos/articulos/pdf1164.pdf

Núñez-Sánchez, R., y Coto-Millán, P. (2012). The impact of public reform on the productivity of Spanish Ports: a parametric distance function approach. Transport Policy, 24(C), 99-108.

Rodríguez-Álvarez, A., y Tovar, B. (2009). Reformas regulatorias $y$ eficiencia en el sector portuario español (Economic Discussion Papers, Efficiency Series Paper 05/2009). Universidad de Oviedo. Dpto. de Economía. https://www.unioviedo. es/oeg/ESP/esp_2009_05.pdf

Rodríguez-Álvarez, A., y Tovar, B. (2012). Have Spanish port sector reform during the last two decades ben successful? A cost frontier approach. Transport Policy, (24), 73-82.

Tovar, B., y Wall, A. (2017). Specialisation, diversification, size and technical efficiency in ports: an empirical analysis using frontier techniques. EJTIR, 17(2), 279-303. https://d1 rkab7tlqy5f1.cloudfront.net/TBM/Over\%20faculteit/Afdelingen/Engineering\%20Systems\%20and\%20Services/EJTIR/Back\%20issues/17.2/2017_02_05\%20 Specialisation\%2C\%20diversification $\% 2 \mathrm{C} \% 20$ size $\% 20$ and $\% 20$ technical\%20 efficiency $\% 20$ in\%20ports\%2C\%20an\%20empirical\%20 D 


\section{Fernando González Laxe}

analysis\%20using\%20frontier\%20techniques. pdf

Villaverde, J., y Fernández-Maza, A. (2012). El Hinterland de las fachadas marítimas españolas. Papeles de Economía Española, (131), 180-199.
https://www.funcas.es/wp-content/uploads/Migracion/Articulos/FUNCAS_PEE/131art14.pdf

Wiegmans, B. (2004). Performance conditions for Container Terminals. Maritime Economics \& Logistics, 6(3), 276-277. 